trächtliche Verluste durch Verflüchtigung herbei; so konnten aus $500 \mathrm{ccm}$ einer $1 \%$ Sublimatlösung nach dem Eindampfen auf $10 \mathrm{ccm}$ anstatt $0,5 \mathrm{nur}$ 0,4608 , beim Eindampfen zur Trockene nur $0,4561 \mathrm{~g} \mathrm{Hg} \mathrm{Cl}_{2}$ analytisch wieder gewonnen werden.

\title{
Notiz zur jodometrischen Bestimmung von Wasserstoffperoxyd.
}

\author{
Von E. Rupp.
}

In Heft 2 pag. 156 des laufend. Jahrganges dieser Zeitschrift berichtete ich über eine jodometr. Bestimmung des Wasserstoffperoxyds der Alkali-Perkarbonate und Persulfate. Wie ich nachträglich bemerkte, ist mir in Bezug auf die Gehaltsbestimmung von WasserstoffperoxydLösungen eine auf das Jahr 1887 zurückdatierende Arbeit von Herrn Professor H. Thoms über "Die Methoden der qantitativen Bestimmung von Wasserstoffsuperoxyd " ${ }^{1}$ ) entgangen. Derselbe unterzieht hierin die bekannten Methoden der Wasserstoffperoxyd-Bestimmung einer vergleichenden Prïfung und empfiehlt gleichzeitig eine jodometrische Bestimmungsweise in ganz ähnlicher Ausführung, wie von mir vorgeschlagen, zur Anwendung. Es muss somit die Priorität genanntem Autor zuerkannt werden.

\section{Zur quantitativen Bestimmung der Alkaloide mittelst titrierter Jodlösung.}

Von M. Scholtz.

(Eingegangen den 1. V. 1900.)

Nachdem ich im vorigen Jahre an dieser Stelle gezeigt habe, dass die von Kippenberger zur quantitativen Bestimmung der Alkaloide vorgeschlagenen Methoden nach Theorie und Praxis unhaltbar sind ${ }^{2}$ ), kommt Kippenberger in einem längeren Anfsatze auf diese Methoden zurück, in welchem er zu dem Schlusse gelangt:

„Nach Feststellung der durch diese Arbeit gegebenen Thatsachen erachte ich die Angaben von Scholtz, welche geeignet erscheinen mussten, meine experimentell so reichhaltigen und nach vielen Richtungen hin ausgedehnten Untersuchungen in ein falsches Licht $\mathrm{zu}$ stellen, für widerlegt ${ }^{* a}$ ).

1) Arch. d. Pharm. 225, 341.

2) Arch. d. Pharm. 1899, 71.

3) Arch. d. Pharm. 1900, 135. 
Durch diese Schlussfolgerung bin ich gezwungen, anf die Kippenberger'schen Methoden nochmals mit wenigen Worten zurückzukommen.

Da es bei der Kippenberger'schen Abhandlung vielfach schwierig ist, aus derselben den Kernpunkt der Frage zu erkennen, so sehe ich mich genötigt, die Hauptpunkte meiner vorjährigen Abhandlung zu wiederholen. Ich hatte die Ergebnisse meiner Versuche in drei Sätze zusammengefasst und will in möglichster Kürze zeigen, dass keiner derselben einer Modifikation bedarf.

1. Die von Herrn Kippenberger seinen analytischen Methoden zu Grunde gelegten theoretischen Anschauungen stehen im Widerspruch mit elementaren chemischen Gesetzen.

Diesen Widerspruch erblickte ich darin, dass Kippenberger zur Er.klärung des Reaktionsverlaufs zwischen Alkaloid- und Jodlösung annimmt, dass Reaktionen, welche sich mit grosser Energie vollziehen, in umgekehrter Richtung verlaufen, was lediglich dadurch bedingt sein soll, dass der hierbei entstehende Körper das Bestreben hat, sich mit einem dritten, in der Lösung vorhandenen Körper zu verbinden. Dass es zur Erklärung der Bildung der Superjodide keiner weittragender Theorien bedarf, sondern dass dieselbe sich nach der einfachen Gleichung vollzieht:

$$
\mathrm{Alk} \cdot \mathrm{HCl}+\mathrm{KJ}+\mathrm{Jx}=\mathrm{Alk} \cdot \mathrm{HJ} \cdot \mathrm{Jx}+\mathrm{KCl},
$$

welche man stets hierfür angenommen hatte, ist zum Ueberfluss neuerdings auch von Gordin experimentell bewiesen worden, welcher auf diese Umsetzung die Methode der quantitativen Bestimmung der Alkaloide unter Anwendung von Phenolphthalein als Indikator gründete ${ }^{1}$ ).

2. Die von Herrn Kip penberger angeführten Analysenzahlen sind zum grössten Teil unter den von ihm innegehaltenen Bedingungen nicht $z u$ erhalten.

Dies bezog sich in erster Linie auf die erste von Kippenberg er veröffentlichte Methode zur Bestimmung der Alkaloide mittelst Jodlösung, durch welche er im Gegensatz zu früheren Versuchen Anderer gezeigt zu haben glaubte, dass durch $1 \mathrm{Mol}$. Alkaloid der Jodlösung stets 3 Atome Jod entzogen werden. Die zur Begründung dieser Methode veröffentlichten Analysenzahlen erklärte Kippenberger nachträglich dadurch, dass er tür jedes Alkaloid eine andere, eigens auf dieses Alkaloid eingestellte Jodlösung angewandt habe, deren Jodkaliumgehalt so gewählt war, dass auf $1 \mathrm{Mol}$. Alkaloid gerade 3 Atome

1) Ber. d. d. chem. Ges. 32, 2871. 
Jod verbraucht wurden ${ }^{1}$ ). In der betreffenden $A$ bhandlung ${ }^{2}$ ) ist indessen ganz eindeutig von der üblichen ${ }^{1} / 20$-Normal-Jodlösung die Rede, es würde mithin hier das Unzutreffende meines Urteils den ungenauen Angaben der sonst sehr ausführlichen Ki p pen berger'schen Abhandlung zur Last fallen.

Die zur Begründung der zweiten Methode, bei welcher die salzsaure Alkaloidlösung mit Jodsilber-Jodkaliumlösung und dann erst mit Jodlösung versetzt wird, von ihm angeführten Analysenzahlen sucht Kippenberger mit meinen Resultaten in Einklang zu bringen, indem er vier seiner Versuche den meinigen gegenüberstellt und zeigt, dass die Resultate nahezu übereinstimmen. Selbst wenn diese Gegenüberstellung richtig wäre, wäre sie ohne Wert. Ich habe ansdrücklich darauf hingewiesen, dass ich bei dieser Methode bei einigen Alkaloiden Resultate erhielt, welche den theoretischen Werten und den von Kippenberger erhaltenen sehr nahe stehen, aber gleichzeitig betont, dass man durch Anwendung wechselnder Mengen von Jodlösung und Silberlösung beinahe jedes beliebige Resultat, also auch das theoretisch richtige, erzielen kann. Um diesen Einfluss der Silberlösung und der Jodlösung, und ferner den der Dauer der Einwirkung der Lösungen zu zeigen, habe ich mit jedem Alkaloid mehrere Versuche ausgeführt, und von diesen führt Kippenberger nur diejenigen an, deren Resultate den seinigen am nächsten stehen, auch wenn sie unter ganz anderen Bedingungen erzielt worden sind, wie die seinigen. Vergleicht man die von Kippenberger und von mir unter Innehaltung derselben Bedingungen erzielten Resultate, so sieht die Gegenüberstellung etwas anders aus:

Es fand ein Verbrauch von ${ }^{1} 20$-Normal-Jodlösung statt bei Anwendung von

\begin{tabular}{|c|c|c|c|c|}
\hline & & Kippen & berger & Scholtz \\
\hline 0,1 Narkotin & . & . . 9,7 & $\mathrm{ccm}$ & $8,4 \mathrm{ccm}$ \\
\hline 0,1 Brucin & & . 12,9 & $n$ & 12 \\
\hline 0,1 Chinin & . & . 24,5 & n & 19,4 \\
\hline 0,1 Morphin & & . 13,4 & ” & 11,9 , \\
\hline
\end{tabular}

Wie man sieht, ist die Verwendbarkeit meiner Versuche zu Gunsten der Kippenberger'schen Methode eine sehr beschränkte.

3. Die von Herrn Kippenberger empfohlenen Methoden zur quantitativen Bestimmung der Alkaloide mittelst titrierter Jodlösung sind unbrauchbar.

1) Ztschr. f. anal. Chem. 38, 282.

2) Ztschr. f. anal. Chem. 34, 317. 
Nach der von Kippenberger gegebenen, oben erwähnten Erklärung bezüglich der ersten Methode wird diese wohl von ihm selbst nicht mehr aufrecht erhalten, und betreffs der anderen genügt es, nochmals auf die Thatsache hinzuweisen, dass das Resultat von vier verschiedenen Faktoren abhängt, nämlich von der Menge des Alkaloids, von der Menge der Jodlösung, von der Menge der Silberlösung und von der Dauer der Einwirkung.

Endlich kommt Kippenberger zu dem Schlusse:

„Dass die Methoden der titrimetrischen Bestimmung der Alkaloide in Form ihrer Jodverbindungen nicht $z u$ den besten analytischen Methoden gehören, gebe ich gerne zu. Ob dieselben gegenüber den anderen bekannten Methoden der quantitativen Bestimmung durch Abscheidung der Alkaloide in unlöslicher Verbindung einen Vorteil gewähren würden, musste mir gleichgiltig sein, da ich es mir zur Aufgabe gestellt hatte, die bei der Einwirkung von Jodlösung auf Alkaloidsalze auftretenden Verhältnisse zu klären, um zu zeigen, dass die bis zur Inangriffnahme meiner Arbeiten litterarisch des öfteren aufgetretene und als solche stets bestätigte Ansicht, dass bei der Einwirkung ron Jod auf Alkaloidsalze glatt 2 Aequivalente Jod auf $1 \mathrm{Mol}$. Alkaloidsalz verbraucht werden, eine unexakte und dementsprechend die im Gebrauch befindliche Bestimmungsmethode der Alkaloide unter Zugrundelegung dieser Zahlen eine unrichtige sei“.

Dann wäre allerdings meine Kritik dieser Methoden und der zu ihrer Begründung angeführten Beispiele nicht erforderlich gewesen. Thatsächlich hat Kippenberger die quantitative Bestimmung der Alkaloide mittelst titrierter Jodlösung in der von ihm ausgearbeiteten Form als exakte analytische Methode veröffentlicht, und zwar in so bestimmter Form, dass der Leser an ihrem Werte nicht zweifeln konnte.- Dass er sich heute über dieselben bescheidener äussert, ist leicht verständlich, falls er indessen trotz der bisherigen Diskussion noch nicht von ihrer völligen Unbrauchbarkeit überzeugt ist, so muss ich darauf verzichten, in wissenschaftlichen Fragen mit ihm zur Uebereinstimmung zu gelangen ${ }^{1}$ ).

1) Nachdem jetzt beide Teile in dieser Frage in entsprechender Weise zu Worte gekommen sind, erachten wir diese Angelegenheit für diese Zeitschrift als erledigt. Redaktion. 